\title{
A novel random discrete element analysis of rock fragmentation
}

\author{
Tao Zhao ${ }^{1}$, Yong $\mathrm{Liu}^{2 *}$ \\ ${ }^{1}$ Department of Civil and Environmental Engineering, Brunel University London, London, UB8 3PH, United \\ Kingdom
}

${ }^{2}$ State Key Laboratory of Water Resources and Hydropower Engineering Science, Institute of Engineering Risk and Disaster Prevention, Wuhan University, 299 Bayi Road, Wuhan 430072, P.R. China.

*Corresponding author: E-mail: liuy203@whu.edu.cn

\begin{abstract}
This paper presents a novel probabilistic approach of random discrete element analysis (RDEA)

10 to investigate the mechanism of rock fragmentation under uniaxial compression. This model combines the advantages of both random field theory and discrete element method in characterizing the spatial variation and uncertainty of microscopic material properties. The numerical results reveal that the stress-strain curves of a group of tests can match well the general trend of the experimental data, with the mean uniaxial compressive strength (UCS) of $1510.18 \mathrm{MPa}$ and the mean Young's modulus of $1.73 \mathrm{GPa}$. The coefficient of variation (COV) for the rock samples is much lower than that of the initial random fields due to the averaging effect of microscopic material property in obtaining the bulk values. The rock fragmentation is initiated by the breakage of weak particles within the rock mass and it develops rapidly as the vertical loading stress approaches the UCS. The final damage zone resides dominantly in the weak region of the rock sample and the distribution of material coefficients follows a similar Beta distribution as the corresponding initial random field. Rock samples with persistent "pillar-like" structures of strong particles can effectively resist the normal compression, resulting in high rock strengths. The traditional DEM simulation with a set of constant material properties can only represent one extreme realization of random field, which could significantly overestimate the rock strength. The proposed RDEA approach can effectively capture the uncertainty and complex interactions of rock fragmentation in a more realistic and reliable way.
\end{abstract}

\section{Keywords}

Random field, DEM, uniaxial compression, brittle failure, rock fragmentation 


\section{Introduction}

Rock fragmentation occurs commonly in some natural events such as rockslides, rockfalls and rock avalanches ${ }^{1-4}$. It has been considered as an important factor governing the extremely high mobility and destructive power of these hazards ${ }^{1,2}$. In general, the fragmentation process can be affected by many factors, such as the local topography, microstructure of rock mass and the loading rate. The related research focus mainly on the mechanism of rock fragmentation and its influence on the runout distance and the involved energy losses of rock fragments, through field observations, laboratory tests and numerical simulations ${ }^{5-7}$.

In the field, the properties of rock can vary significantly at different locations due to the variability of constituent material properties and the formation history, which should intuitively be considered as spatial variables. The complex spatial microstructure of rock can affect its

40 geotechnical properties, such as discontinuity, inhomogeneity, anisotropy, and nonlinear elasticity. For example, under external loading, the damage of rock is likely to be initiated at weak regions as the locally concentrated stress can exceed the material strength easily. However, quite few studies have considered the spatial heterogeneity of rock properties, though it is common in reality (e.g. Qin and $\mathrm{Chian}^{8}$ ). In numerical analysis, the rock properties can be modelled as multi-dimensional and multi-variate random fields. The concept of random field can thus be used to characterize the non-uniformity of material properties in a consistent manner (e.g. Fenton and Griffiths ${ }^{9,}$ Liu et al. ${ }^{10}$, Liu et al. ${ }^{11}$, Casagrande et al. ${ }^{12}$ ).

On the other hand, the discontinuity of rock mass can be conveniently investigated by the discrete element method (DEM). It has been employed widely to analyze the brittle failure of

50 solid materials, e.g. rock fragmentation ${ }^{13,14}$, particle crushing ${ }^{15,16}$ and damage of cemented geomaterials $^{7,17}$, due to its capability of modeling the complex brittle responses of solid mass under external loadings, such as crack initiation and propagation ${ }^{18,19}$. At failure, the internal fractures propagate and nucleate rapidly within the solid mass due to the breakage of interparticle cementations (i.e. bonds). It can be induced by excessive compressive, tensile or shear loading, together with the transmission and reflection of stress waves at impact ${ }^{2,20}$. The characteristics and energy dissipation mechanisms of solid fragmentation (e.g. fracturing stress, 
size and number of fragments) depend primarily on the material shear strength and loading strain rate ${ }^{21}$.

In this research, in order to investigate the non-uniformity of granular properties, the random field theory has been integrated into DEM, such that the fragmentation of a heterogeneous rock mass can be readily investigated. The integrated model of random field and DEM is hereafter termed random discrete element analysis (RDEA). The RDEA has been employed to analyse the mechanical responses of rock mass during the fundamental uniaxial compression tests, including the stress-strain relationship and the brittle failure pattern. The Monte-Carlo simulation scheme is used in RDEA to run 500 simulations with spatially randomly distributed material properties (e.g. Young's modulus, bonding strength and friction coefficient). By doing so, the overall performance of the rock sample can be examined in a statistical manner.

\section{Methodology and Model Configuration}

\section{$2.1 \mathrm{DEM}$ model}

The integrated RDEA model has been developed by programming the random field generator in the open source DEM code ESyS-Particle ${ }^{22}$. In the DEM model, the interactions between bonded particles are calculated using the parallel bond model (PBM) $)^{23}$, as:

$$
F_{b n}=K_{b n} \cdot \Delta u_{n}
$$

$$
\begin{gathered}
F_{b s}=K_{b s} \cdot \Delta u_{s} \\
M_{b}=K_{b} \cdot \Delta \theta_{b} \quad M_{t}=K_{t} \cdot \Delta \theta_{t}
\end{gathered}
$$

where $F_{b n}, F_{b s}$ are the normal and shear bonding forces; $M_{b}$ and $M_{t}$ are the bending and twisting moments, respectively; $K_{b n}, K_{b s}, K_{b}$ and $K_{t}$ are the corresponding bonding stiffness, $\Delta u_{n}, \Delta u_{s}$, $\Delta \theta_{b}$ and $\Delta \theta_{t}$ are the relative displacements between the bonded particles in the normal, shear,

80 bending and twisting directions, respectively. The values of relative displacements are 
computed by unit quaternions for spatial rotations of the two $3 \mathrm{D}$ rigid spheres ${ }^{24,25}$. Note that $\Delta u_{n}$ is the difference between the current and initial (prior to the motion onset) distances of the two particles.

The criterion of bond breakage is given as the combined loads exceeding the bonding strength capacity $22,23,26$,

$$
\frac{F_{b n}}{F_{\text {bnMax }}}+\frac{F_{b s}}{F_{b s \operatorname{Max}}}+\frac{M_{b}}{M_{b \operatorname{Max}}}+\frac{M_{t}}{M_{t \text { Max }}} \geq 1
$$

where $F_{b n M a x}, F_{b s \text { Max }}, M_{b \text { Max }}$ and $M_{t \text { Max }}$ are the bond strengths in the normal, shear, bending and twisting directions, respectively. The parallel bond model has been calibrated comprehensively in our recent publication, Zhao et al. ${ }^{27}$.

Once the bond breaks, the particles become dispersed and they interact with each other via the linear-elastic spring contact model, as

$$
\begin{gathered}
F_{n}=K_{n} \cdot u_{n} \\
F_{s}^{i}= \begin{cases}F_{s}^{i-1}+K_{s} \cdot \Delta u_{s}, & \text { if } F_{s}^{i-1}<\mu F_{n} \\
\mu F_{n}, & \text { if } F_{s}^{i-1} \geq \mu F_{n}\end{cases} \\
M=F_{s}^{i} \times \boldsymbol{r}
\end{gathered}
$$

where $F_{n}, K_{n}$ and $u_{n}$ are the normal contact force, stiffness and overlapping distance between two particles in contact; $F_{s}^{i}$ and $F_{s}^{i-1}$ are shear forces calculated at the current and previous simulation time steps, respectively; $\Delta u_{s}$ is the corresponding incremental shear displacement; $K_{s}$ is the shear stiffness; $\mu$ is the friction coefficient. $\boldsymbol{M}$ is the shear induced moment; $\boldsymbol{r}$ is the vector linking the center and contact point between the two particles.

100 Figure 1(a) shows the configuration of uniaxial compression test on a rock sample in DEM. In the test, an assembly of 31,400 densely packed spherical particles are generated within a rectangular parallelepiped domain. The particle radius distributes uniformly in a narrow range 
of [0.75 mm, $1.5 \mathrm{~mm}]$, instead of a constant size, which imposes a necessary size randomness as it contains a variety of microscopic particle properties. These particles are cemented together by the parallel bond model to represent an intact solid rock mass. The bulk density $(\rho)$ of the rock mass is $1379 \mathrm{~kg} / \mathrm{m}^{3}$, as determined by the individual particle density $\left(\rho_{s}=2650 \mathrm{~kg} / \mathrm{m}^{3}\right)$ and packing porosity $(n=0.48)$. The particle Young's modulus $\left(E_{p}\right)$, bonding strength $(c)$ and friction coefficient $(\mu)$ are set as random values based on the random field theory (see Section 2.2). In the DEM simulations, a small damping coefficient of 0.01 is applied at particle contacts to account for the energy dissipation by shearing off particle asperities and plastic deformations. The damping coefficient is determined by trial and error to ensure it has negligible influence on the overall dynamics of the rock sample. The choice is in line with the DEM model configurations in Zhao et al. ${ }^{27}$ and Zhao et al. ${ }^{28}$. The numerical iteration time step $(\Delta t)$ is set as small as $2 \times 10^{-7} \mathrm{~s}$ to guarantee numerical stability. The vertical loading was applied by 115 controlling the axial deformation of the rock sample at a constant strain rate of $1 \mathrm{~s}^{-1}$.
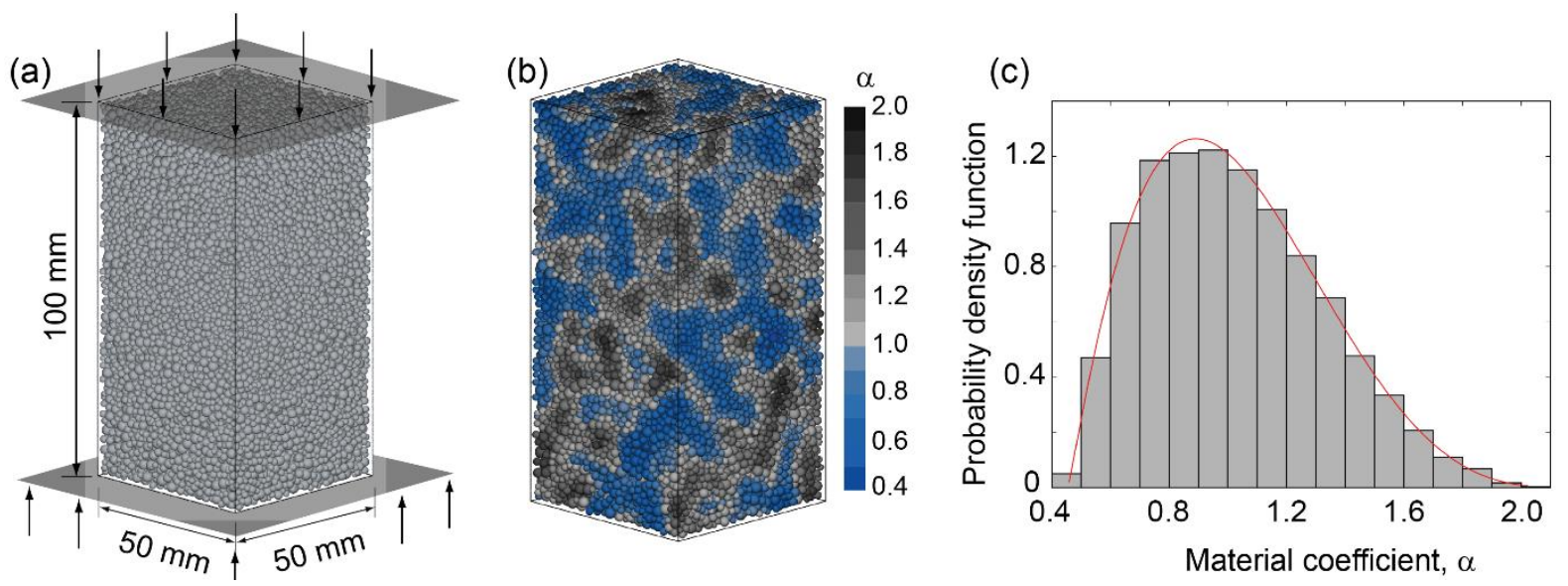

Figure 1. (a) DEM model configuration of uniaxial compression test; (b) a sample of random field generated for the rock sample; (c) the frequency distribution of the material coefficient for (b). The red curve represents the target Beta distribution, with the parameters $a=1.86, b=3.78$, mean $=1$ and coefficient of variation $=0.3$.

\subsection{Random field theory}

This study employs the discrete random field, in which the material properties are evaluated as random variables at discrete points. In the random field, all points are mutually correlated by a specified correlation length (i.e. scale of fluctuation). The Young's modulus, 
125 bonding strength and friction coefficient are herein considered as spatially non-uniform. A standard Gaussian random field is first generated by the modified linear estimation method ${ }^{10}$, which provides an efficient approach to simulate a 3D Gaussian random field with a squared exponential auto-correlation function. The standard Gaussian random field is then transformed into Beta random fields for these three variables with the statistical parameters prescribed in 130 Table 1. The probability density function (PDF) for the standard Beta distribution $(X \sim \operatorname{Beta}(a, b))$ is given as,

$$
f(x)= \begin{cases}\frac{1}{B(a, b)} x^{a-1}(1-x)^{b-1} & \text { if } 0<x<1 \\ 0 & \text { otherwise }\end{cases}
$$

where $B(a, b)=\int_{0}^{1} x^{a-1}(1-x)^{b-1} d x, a$ and $b$ are shape parameters. For a Beta variable whose bounds are not 0 and 1, two additional statistics are needed to determine the bounds. For instance, the statistical mean value and coefficient of variation (COV) are adopted in this study. Here, $\mathrm{COV}$ is defined as the ratio between the standard deviation and the mean.

Table 1. Prescribed statistical parameters for variables with spatial non-uniformity. Note: the shape parameters for the Beta distribution are selected following the field data reported in Liu et al. ${ }^{29}$. Without loss of generality, a set of typical values for coefficient of variation and scale of fluctuation are adopted.

\begin{tabular}{|c|c|c|c|c|}
\hline Variable & Distribution & Mean & $\begin{array}{c}\text { Coefficient of } \\
\text { variation }\end{array}$ & $\begin{array}{c}\text { Scale of } \\
\text { fluctuation }\end{array}$ \\
\hline $\begin{array}{c}\text { Young's } \\
\text { modulus }\end{array}$ & $X \sim \operatorname{Beta}(1.86,3.78)$ & $3.1 \mathrm{GPa}$ & 0.3 & $0.01 \mathrm{~m}$ \\
\hline $\begin{array}{c}\text { Bonding } \\
\text { strength }\end{array}$ & $X \sim \operatorname{Beta}(1.86,3.78)$ & $13 \mathrm{MPa}$ & 0.3 & $0.01 \mathrm{~m}$ \\
\hline $\begin{array}{c}\text { Friction } \\
\text { coefficient }\end{array}$ & $X \sim \operatorname{Beta}(1.86,3.78)$ & 0.577 & 0.3 & $0.01 \mathrm{~m}$ \\
\hline
\end{tabular}

In the analysis, a mapping is established between the coordinates of discretized particles and the generated random fields, as such, the value of random field at the individual particle centre is set as the material coefficient $(\alpha)$ of that particle. The exact material properties are obtained by multiplying the material coefficient by the mean material properties (i.e. $E_{p 0}, c_{0}$, $\left.\mu_{0}\right)$. These mean values are calibrated based on trial and error, such that the DEM results of uniaxial compression test on a uniform sample can roughly match the experimental data. The 
random field generated on the rock sample is illustrated in Figure 1(b), with the material coefficient following the Beta distribution (see the distribution in Figure 1(c)). In this approach, the mean Young's modulus, bonding strength and friction coefficient are prescribed values, while other statistical parameters (e.g. COV, and scale of fluctuation) remain unchanged for a

150 series of tests. Following the Monte-Carlo simulation scheme, the uniaxial compression test on the same granular assembly, but with spatially randomly distributed material properties, can be repeated for a number of times, as the random field of the rock sample is different from one simulation to another. In this study, 500 simulations have been performed to statistically analyse the performance of the rock sample.

This proposed RDEA approach has the apparent advantage in modelling the mechanical behaviour of a specific type of rock when compared with the traditional DEM simulations using only one set of constant microscopic material properties. The RDEA considers the spatial variation of material properties through a series of different random fields, while the mean material properties remain constant for different simulations. Through statistical analysis, the

160 simulations can reproduce the overall mechanical behaviour of rock mass with similar internal structures, e.g. rock samples collected at the same site. Thus, this research can effectively capture the uncertainty and complex interactions of a specific type of granular material in a more realistic and reliable way.

\section{Results}

Figure 2 illustrates the spread of stress-strain curves of uniaxial compression tests by RDEA for 500 repeated simulations on rock samples with different random fields. The experimental data on coal rock samples with parallel and normal stratigraphic beddings by Liu et al. ${ }^{30}$ are also presented for comparison purpose. The experiments employed a much smaller loading strain rate $\left(0.01 \mathrm{~s}^{-1}\right)$ than the DEM tests $\left(1 \mathrm{~s}^{-1}\right)$. However, under the quasi-static loading

170 condition, the difference in stress-strain relationship is quite small ${ }^{31}$. Thus, the comparison can be regarded as reliable. In the figure, the minimum $\left(0^{\text {th }}\right.$ percentile $), 5^{\text {th }}$ percentile, median $\left(50^{\text {th }}\right.$ percentile) and maximum $\left(100^{\text {th }}\right.$ percentile) of the uniaxial compressive strength (UCS, the 
peak stress on the stress-strain curve) of these tests are also highlighted by various colors. According to the figure, the numerical results exhibit a wide distribution which match well the general trend of the experimental data. Thus, the general mechanical behavior of the type of coal rock shown in Figure 2 can be well captured by the set of random discrete element analyses. After failure, the numerical samples show apparent brittle behavior with sudden drops of stresses, while the experimental data exhibits a more ductile responses with gradual decrease of normal stresses. The traditional DEM simulation with a set of constant material properties 180 (i.e. the uniform sample in Figure 2) has the upper bound of stress for all possible testing results. It has the UCS of 11.9 MPa which is higher than the one (10.2 MPa) obtained in Zhao et al. ${ }^{27}$. The difference is due mainly to the much narrower particle grading used in the current study.

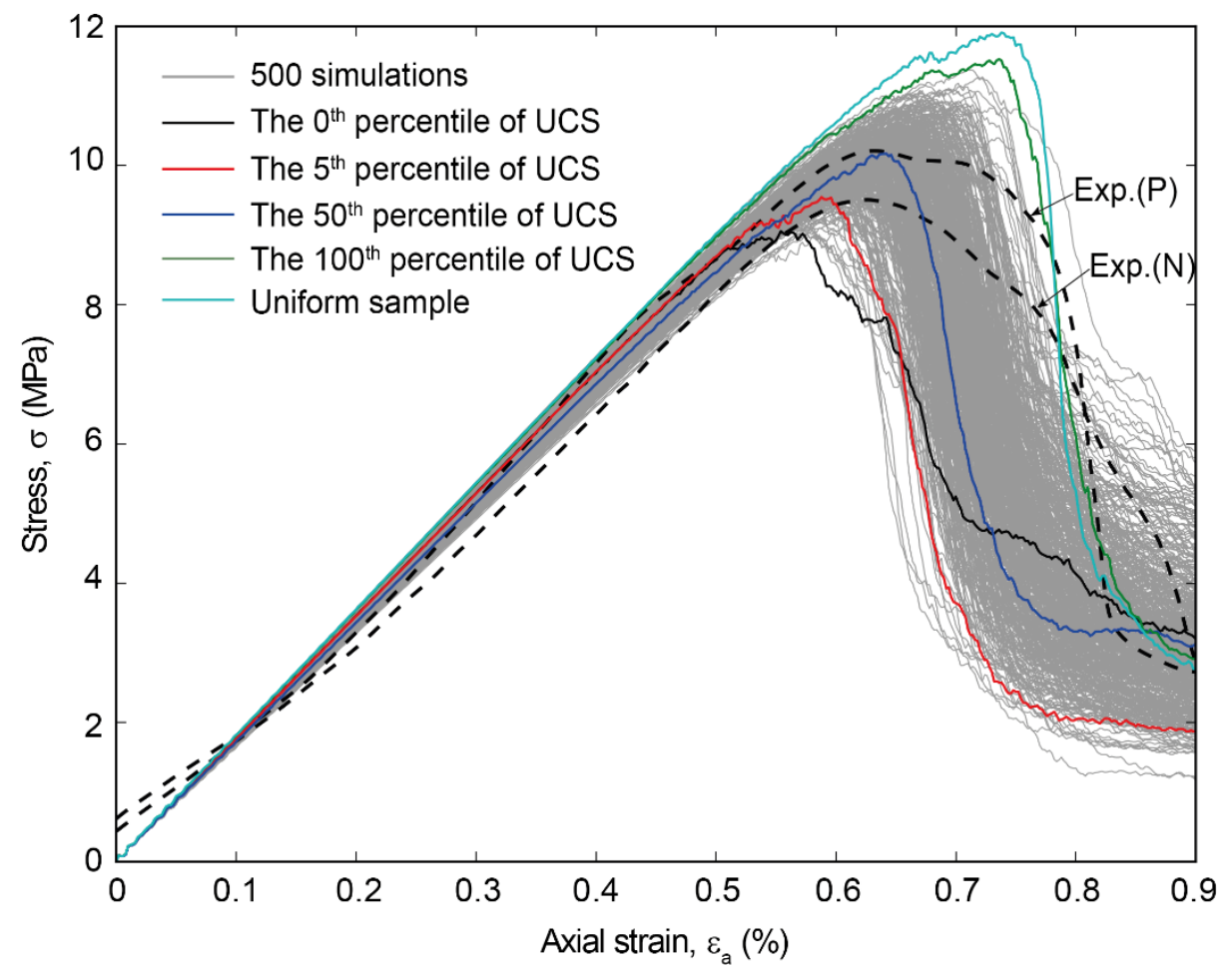

Figure 2. The stress-strain relationship of uniaxial compression test on rock samples with a constant vertical loading strain rate of $1 \mathrm{~s}^{-1}$. "Exp.(P)" and "Exp.(N)" stand for experimental data of uniaxial compression tests on coal rock with parallel and normal beddings to the loading direction, respectively (the strain rate used in experiments is $0.01 \mathrm{~s}^{-1}$ ).

According to Figure 2, and the Young's modulus of rock (E) is calculated as the gradient of the initial linear portion (with strain up to $0.3 \%$ ) of the stress-strain curve. The histograms 190 of UCS and $\mathrm{E}$ in 

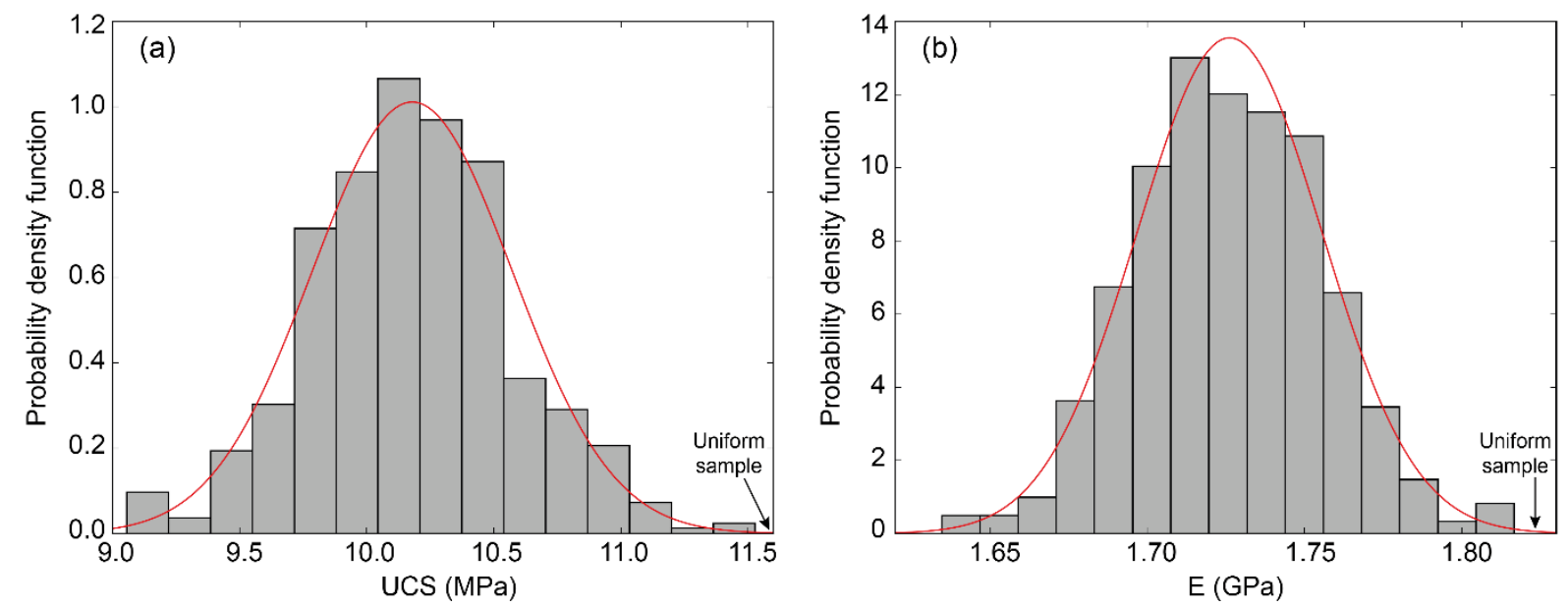

Figure 3 (a) and (b) show that for the 500 independent DEM tests, the UCS and Young's modulus of the rock sample generally follow the normal distributions. The UCS has the average value of $10.18 \mathrm{MPa}$ and the COV of 0.038 . The bulk Young's modulus has the average value of $1.73 \mathrm{GPa}$ and the COV of 0.017 . The average UCS and bulk Young's modulus of rock samples are much lower than that of the uniform sample, indicating the uniform material property assumption, as traditional DEM simulation does, can significantly overestimate the rock strength and bulk Young's modulus. The COVs in UCS and Young's modulus are significantly lower than the corresponding values of the random fields $(0.3)$ due to the averaging effect on the variability of microscopic properties.
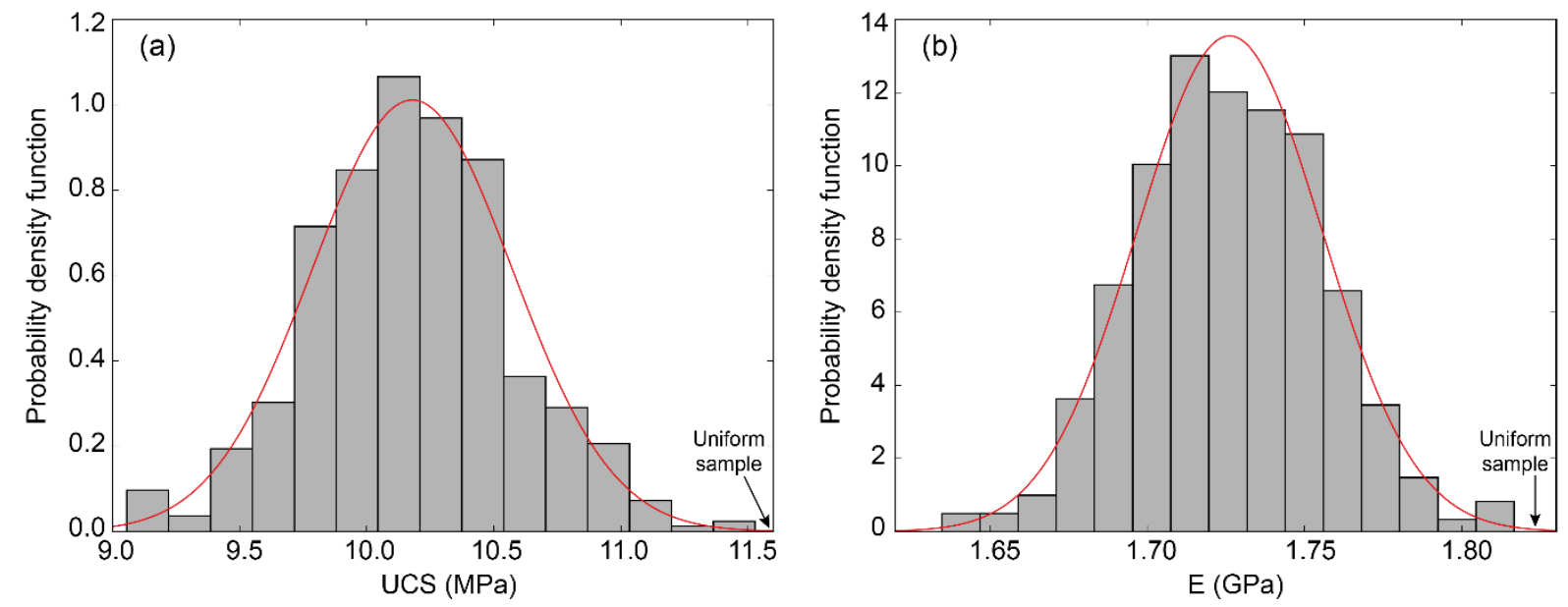

Figure 3. Histograms of the UCS and Young's modulus of the rock sample. The red curves show normal distributions of the data. The results of DEM tests on the uniform sample are labeled on the figure. 


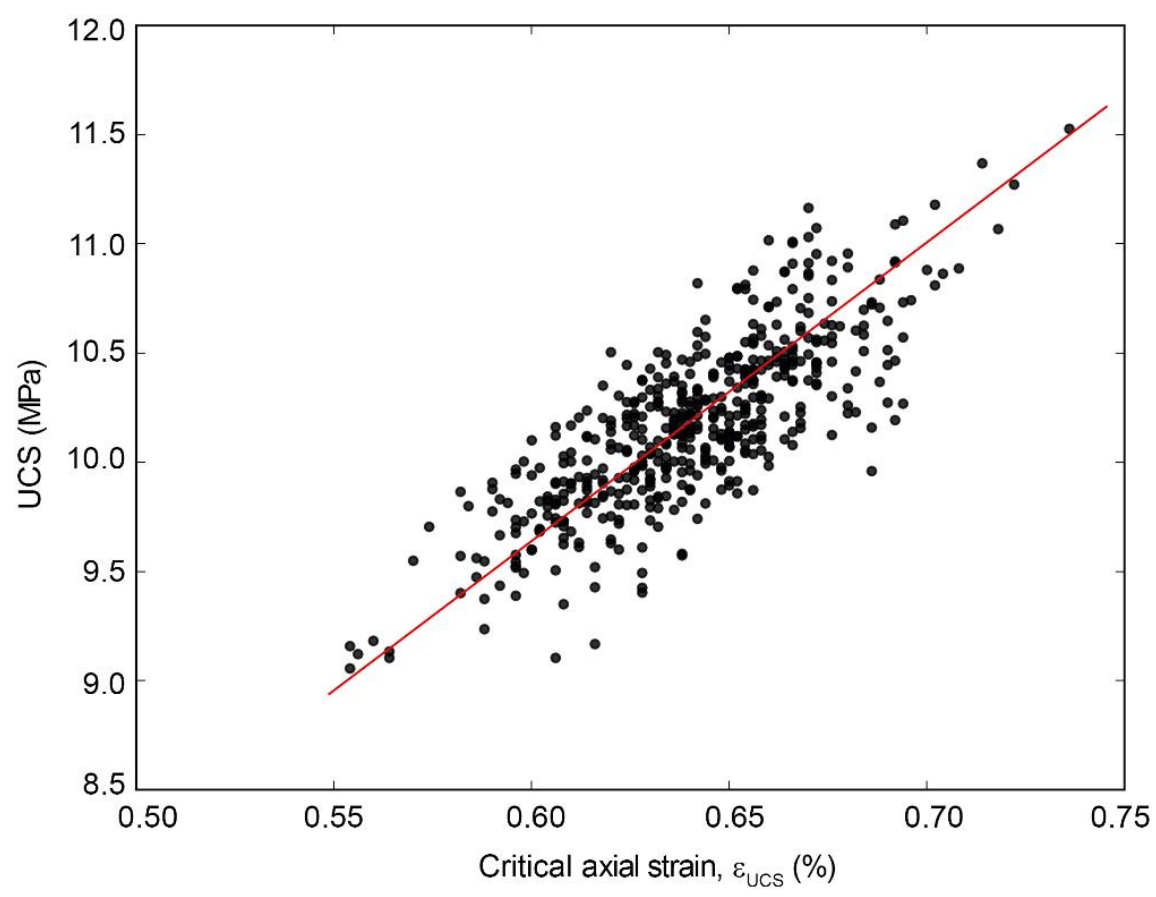

205 Figure 4. The UCS and the corresponding axial strain ( $\left.\varepsilon_{\mathrm{UCS}}\right)$ of the 500 simulations. The red fitting line shows the general trend of the distribution.

Figure 2 also shows that despite the variance, the corresponding axial strain of each test when the UCS is reached increases with UCS. This is summarized as the relationship between the UCS and the critical axial strain $\left(\varepsilon_{U C S}\right)$ in Figure 4 . The general trend shows that the UCS increases with the critical axial strain, with the dominant frequency of $\varepsilon_{U C S}$ in the range of $0.6 \%$ to $0.7 \%$. This indicates that the extremely weak and strong rock mass were rarely generated by the proposed randomly field theory. Rock mass of UCS as low as $9.0 \mathrm{MPa}$ can fail easily at very small critical axial strain $(\approx 0.55 \%)$, while rock mass of UCS as high as $11.5 \mathrm{MPa}$ can resist much larger deformation $\left(\varepsilon_{U C S} \approx 0.74 \%\right)$.

215 Figure 5(a) illustrates the evolution of rock damage ratio for the 500 simulations during the uniaxial compression tests. Here, the rock damage ratio is defined as the ratio of the number of bonds broken during the compression to the total number of bonds in the initial sample. According to the figure, the internal rock damage occurs when the axial strain exceeds $0.3 \%$. Then, the bond damage accumulates gradually as the axial strain increases (see the inset plot of Figure 5(a)). A complete analysis of all 500 simulations on the stress level during the fracturing process indicates that the fracture initiation starts at about 0.4-0.68 times of the UCS in each test. This result can match well the experimental data in Cai et al. ${ }^{32}$ that the fracturing 
stress level is around 0.3-0.5 times of the UCS. The rock damage ratio increases rapidly once the axial strain exceeds a threshold value (see the axial strains marked by dashed lines on Figure 225 5(a)). This threshold value corresponds to the critical axial strain discussed in Figure 4. The rapid increase of rock damage is attributed to the sudden release of strain energy as the rock structure can no longer resist the normal loading, which facilitates the propagation and nucleation of internal rock damage zones. For different tests, rock samples of high UCS is fractured at much higher axial strain than those of low UCS, indicating that the strong rock

230 mass can better resist the normal compressions. The final damage ratio of each test is not directly correlated with each other as it is affected by many factors such as the heterogeneity of rock, the orientation of failure plane and the amount of released energy. 

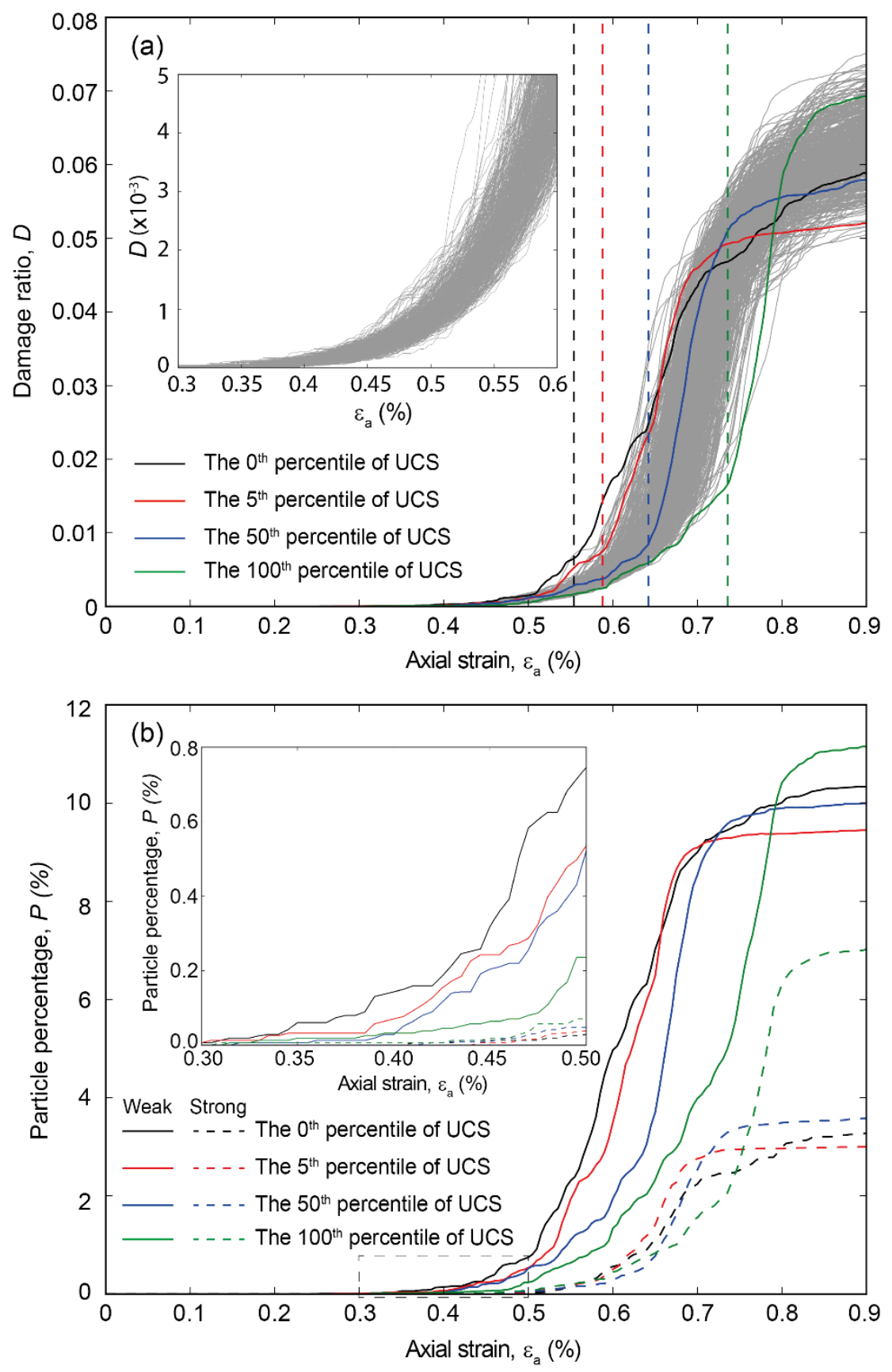

Figure 5. (a) The relationship between the internal rock damage ratio and sample deformation. The damage ratio is defined as the number of bonds broken during the compression over the total number of bonds in the initial rock sample. The dashed line marks the axial strain when the UCS is reached.

(b) The percentage of weak and strong particles in the damage zone. The solid curves represent the percentage of weak particles $(\alpha<1)$, while the dashed curves represent the percentage of strong particles $(\alpha>1)$. Here, the $0^{\text {th }}$ and $100^{\text {th }}$ percentiles represent the tests of the minimum and maximum UCS values among the 500 tests.

Figure 5(b) reports a detailed analysis of the evolution of the percentage of "weak" and "strong" particles in the damage zone for four representative rock samples with the UCS values of the $0^{\text {th }}, 5^{\text {th }}, 50^{\text {th }}$ and $100^{\text {th }}$ percentiles of distribution in 

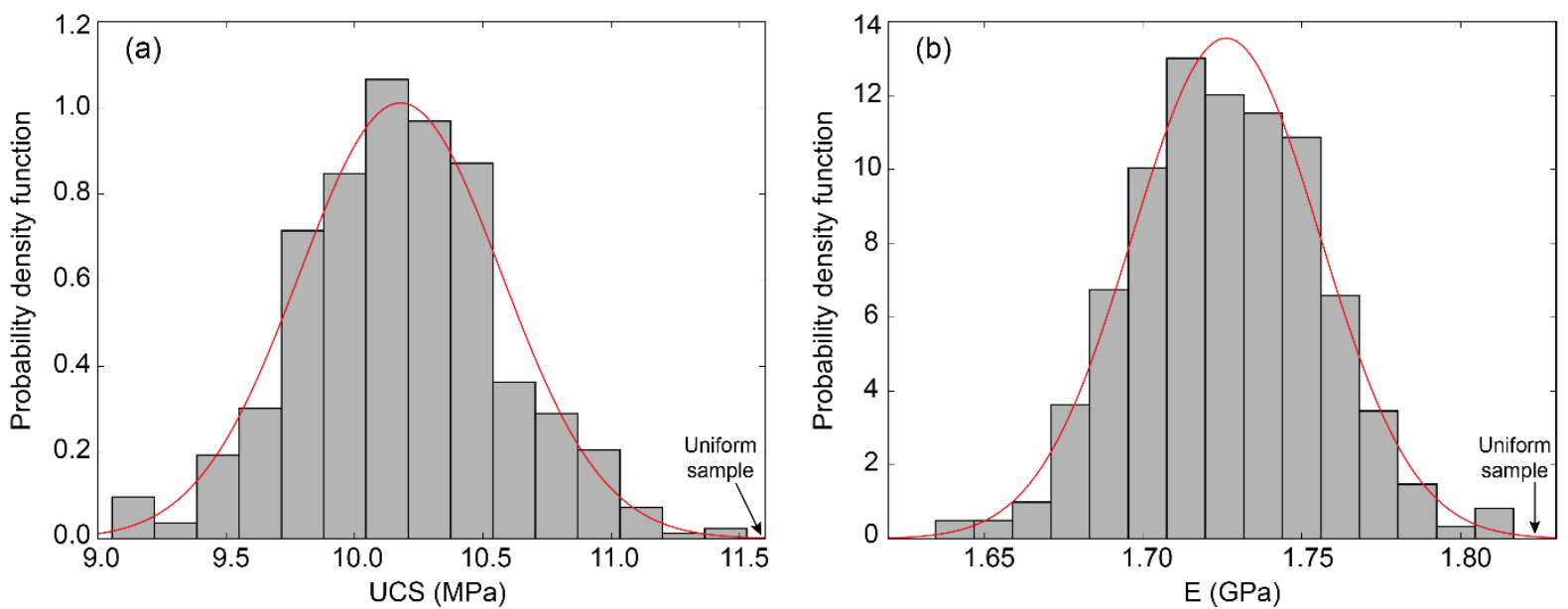

Figure 3(a). Here, the "weak" particle refers to the solid particle of material coefficient smaller than or equal to $1(\alpha \leq 1)$, while the "strong" particle refers to the solid particle of material coefficient larger than $1(\alpha>1)$. In the analysis, only particles with broken bonds (hereafter termed "dispersed particle") are considered as rock fragments located in the damage zone. Figure 5(b) illustrates that the rock damage was initiated by the breakage of weak particles within the rock mass. After initiation, the percentage of dispersed weak particles increases much faster than that of strong particles. Similar to Figure 5(a), after the rock sample reaches the UCS, the percentage of dispersed particles increases rapidly with very small axial deformations. It should be noted that since part of the strong particles are attached to the weak ones, the exact percentage of strong particles in the damage zone should be lower than the results shown on Figure 5(b).

(a) $\varepsilon_{\mathrm{a}}=0.45 \%$

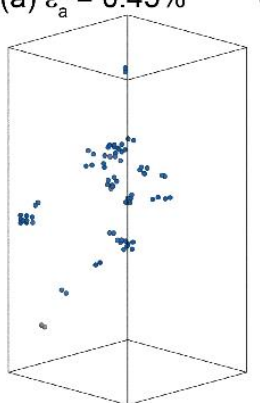

(b) $\varepsilon_{a}=0.5 \%$

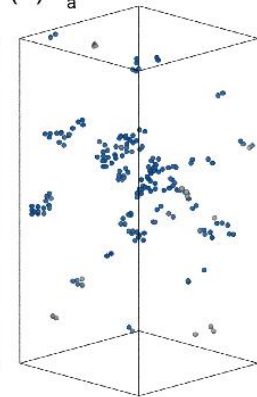

(c) $\varepsilon_{\mathrm{a}}=0.55 \%$

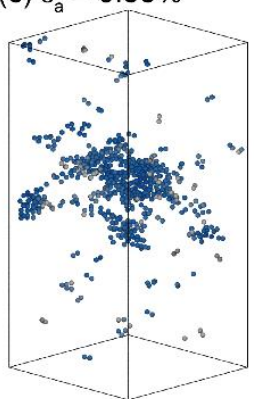

(d) $\varepsilon_{\mathrm{a}}=0.6 \%$

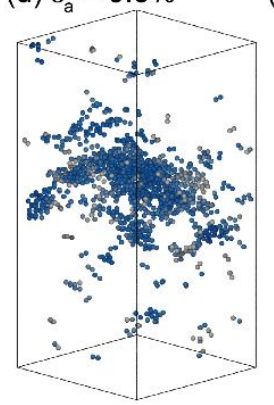

(e) $\varepsilon_{\mathrm{a}}=0.65 \%$

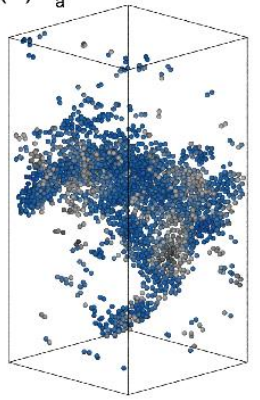

(f) $\varepsilon_{\mathrm{a}}=0.7 \%$

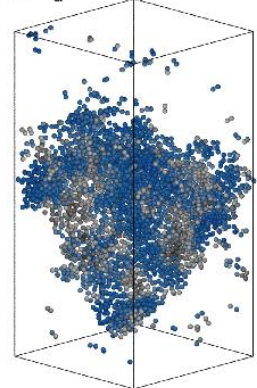

\begin{tabular}{lllllllll}
0.4 & 0.6 & 0.8 & 1.0 & 1.2 & 1.4 & 1.6 & 1.8 & 2.0 \\
\hline
\end{tabular}

Material coefficient, $\alpha$

Figure 6. Evolution of internal damage zone (dispersed particles) of the rock sample with the $5^{\text {th }}$ percentile of UCS. In the figure, the color is set to scale with the value of material coefficient $(\alpha)$. The material coefficient of 1 represents the average value of material properties. 
UCS is shown in Figure 6. According to the plot, the internal rock damage starts at the weak areas (dark blue particles in Figure 6(a)) in the middle of the sample. As the axial strain increases, weak particles break within the initial intact sample and gradually nucleate to form a localized damage zone. In this process, a few strong particles (colored grey) also break (see the percentage in Figure 5(b)). The portion of strong particles in the damage zone increases dramatically at large axial deformations when the rock sample is close to fail (see Figure 6(d, e)). After failure (Figure $6(\mathrm{f}), \varepsilon_{\mathrm{a}}=0.7 \%$ ), an inclined failure plane consisting of a mixture of weak and strong particles can be clearly observed within the sample. This failure plane splits the rock sample into two major wedge-shaped rock fragments.
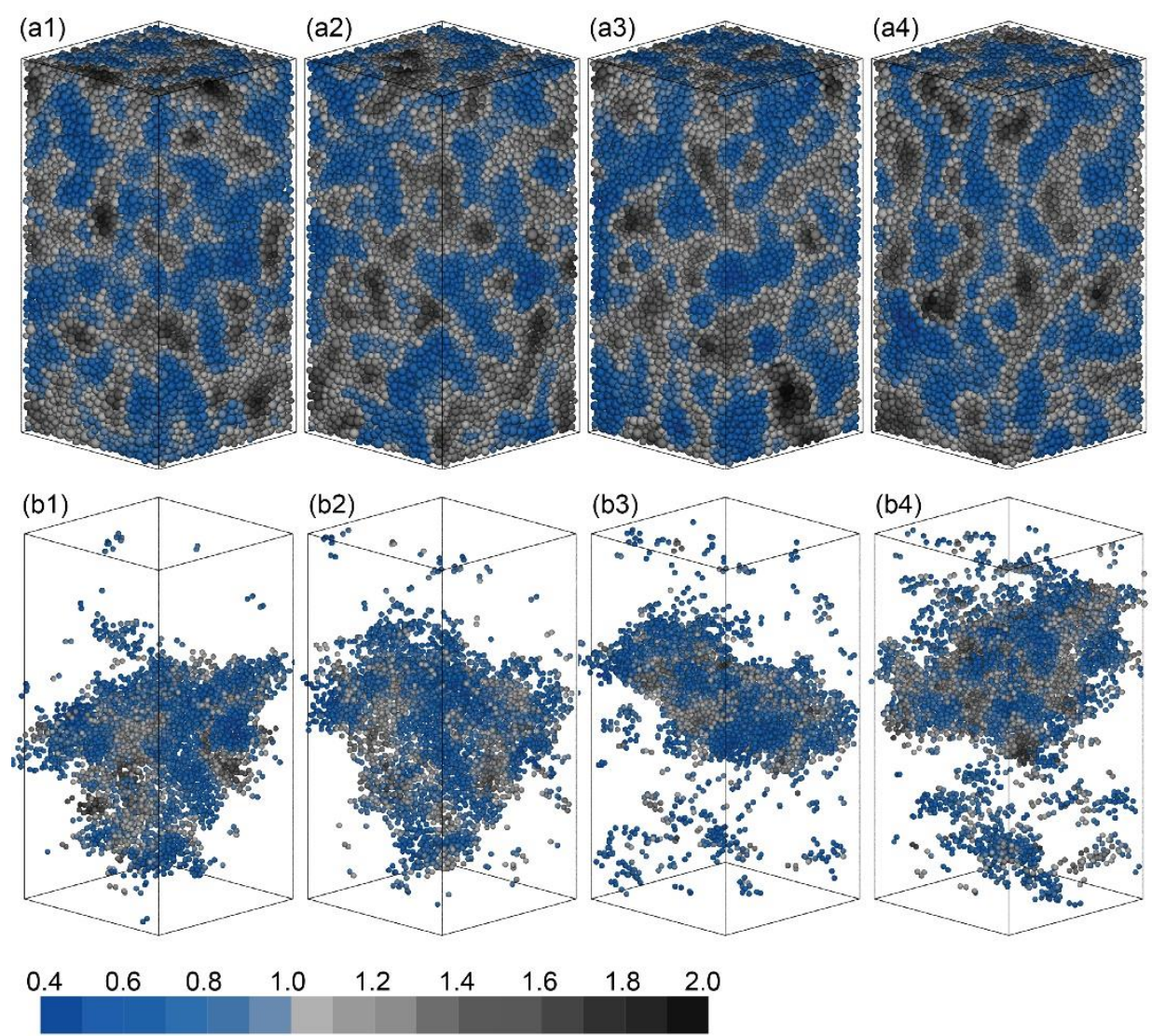

Material coefficient, $\alpha$

Figure 7. Series (a): the random field of the initial rock sample for tests of (a1) the $0^{\text {th }}$ percentile of UCS; (a2) the $5^{\text {th }}$ percentile of UCS; (a3) the $50^{\text {th }}$ percentile of UCS; (a4) the $100^{\text {th }}$ percentile of UCS. Series (b): the final damage zone within the rock sample after the compression for tests of (b1) the $0^{\text {th }}$ percentile of UCS; (b2) the $5^{\text {th }}$ percentile of UCS; (b3) the $50^{\text {th }}$ percentile of UCS; (b4) the $100^{\text {th }}$ percentile of UCS.

Figure 7 illustrates the initial random field and the final damage zone of rock samples with 
different final UCS. As the UCS increases, the internal damage zone becomes gradually flat and locates closer to the upper region of the sample. For the rock sample shown in Figure 7(a1), the strong particle regions are relatively small and disconnected, which are interbedded by the large weak zones. The weak particle regions are particularly dominant in the middle of the sample with inclined orientations. This distribution pattern is favored by the shear failure along an inclined plane during the uniaxial compression test on the rock sample, resulting in very low overall rock strength. On the other hand, for the rock sample shown in Figure 7(a4), the strong and weak particle regions are interbedded with connected pillar-like distributions along the vertical direction. This distribution pattern can effectively resist the normal compression as the strong particle pillars can resist very high normal stresses. The final failure occurs when these particle pillars are crushed under high normal loading stresses, resulting in a relatively flat final damage zone.
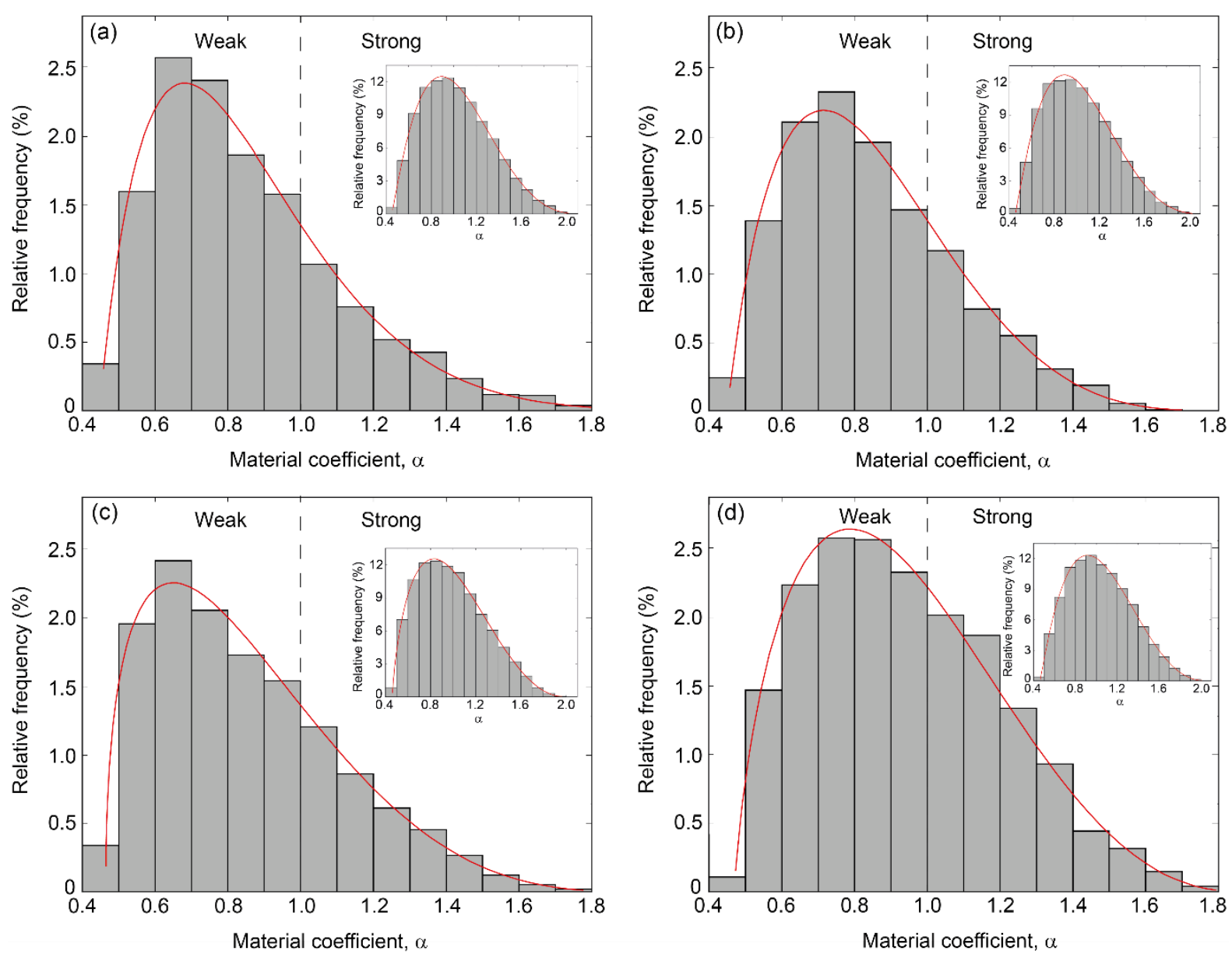

Figure 8. Statistics of material coefficients for particles located in the final damage zone. The frequency is represented by the relative percentage of dispersed particles with different material 
coefficient in the model. (a) test of the $0^{\text {th }}$ percentile of UCS; (b) test of the $5^{\text {th }}$ percentile of UCS; (c) test of the $50^{\text {th }}$ percentile of UCS; (d) test of the $100^{\text {th }}$ percentile of UCS. The red curves represent the best Beta fitting lines. The inset plots are the frequency distribution of material property of the corresponding initial sample.

The distribution of material coefficients for dispersed particles in the damage zone is shown in Figure 8 . Here, the relative frequency is expressed as the percentage of particles in each material coefficient range (bin size) out of the total number of particles in the DEM model. According to the plots, the frequency of material coefficients of dispersed particles all follows the Beta distribution pattern, with similar shape to the corresponding distribution of the initial random field (see the inset plots). The accumulative percentage of dispersed particles increases with the rock strength, indicating that the failure of strong rock will break more particles than that of weaker ones. For each test, the percentage of dispersed weak particles $(\alpha<1)$ is much higher than the strong particles $(\alpha>1)$, and the mean value of material coefficient in the distribution is around 0.7 . This distribution pattern indicates that the final damage zone resides dominantly in the weak region of the sample.

\section{Conclusions}

The fragmentation of rock mass has been investigated by a novel probabilistic approach of random discrete element analysis. The proposed numerical model has integrated the random

310 field theory with the discrete element method, enabling it to analyze the detailed fracturing mechanism of rock mass. The DEM parallel bonded-particle model employs the unit quaternions to evaluate the complex spatial finite rotations and interactions of bonded particles concisely and reliably. The random fields consider the spatial variation of material properties as Beta distribution, while the mean material properties remain constant for different 315 simulations.

The uniaxial compression tests on 500 rock samples investigated in this research can reproduce the general mechanical behaviors of a specific type of rock materials (with distribution $X \sim \operatorname{Beta}(1.86,3.78))$ with the mean UCS of $10.18 \mathrm{MPa}$ and the mean bulk Young's modulus (E) of $1.73 \mathrm{GPa}$. The coefficient of variation (COV) for the rock samples 
320 (0.038 for UCS and 0.017 for E) is much lower than that of the initial random fields $(0.3)$ for the granular particles due to the averaging effect of microscopic material property in obtaining the bulk values. The overall performance of a rock sample with spatially heterogeneous properties is dominated by the weak particle regions, and thus the traditional DEM simulation using constant material properties can significantly overestimate the strength and bulk Young's modulus of the rock sample. The rock damage is initiated by the breakage of inter-particle bonds in weak particle regions within the rock mass and it starts to increase rapidly as the vertical loading stress approaches the UCS. The final damage zone resides dominantly in the weak particle zone of the sample and the distribution of their material coefficients follows a similar Beta distribution as the initial random field. For the weak samples, the weak particle regions are dominantly distributed in the middle of the sample, which facilitate the formation of shear zone along an inclined plane under even low loading stress. For the strong samples, the strong particle regions are connected to form vertical solid "pillars", which can effectively resist the normal compression.

The proposed random discrete element analysis can generate a series of rock samples with 335 the same granular structures, but spatially varied microscopic material properties, e.g. rock samples collected at the same site. Thus, this study can capture the uncertainty and complex interactions of rock fragmentation in a more realistic and reliable way than the traditional DEM modelling approach using only one set of constant material properties. With proper calibrations, the proposed numerical model can significantly extend the applicability of DEM in modelling the granular mechanics.

\section{Acknowledgements}

This research was supported by the National Natural Science foundation of China (Grant No. 51879203) and the open funding of the State Key Laboratory of Water Resources and Hydropower Engineering Science (Wuhan University) (No. 2019SGG02). 


\section{References}

1. De Blasio, F. and G.B. Crosta, Fragmentation and boosting of rock falls and rock avalanches. Geophys. Res. Lett., 2015. 42(20): p. 8463-8470.

2. Crosta, G.B., P. Frattini, and N. Fusi, Fragmentation in the Val Pola rock avalanche, Italian Alps. J. Geophys. Res. Earth Surf., 2007. 112(F1): p. F01006.

350 3. Legros, F., The mobility of long-runout landslides. Engineering Geology, 2002. 63(3-4): p. 301331.

4. Locat, P., R. Couture, S. Leroueil, J. Locat, and M. Jaboyedoff, Fragmentation energy in rock avalanches. Canadian Geotechnical Journal, 2006. 43(8): p. 830-851.

5. Zhao, Y.X., S.M. Liu, G.F. Zhao, D. Elsworth, Y.D. Jiang, and J.L. Han, Failure mechanisms in coal: Dependence on strain rate and microstructure. J. Geophys. Res.: Solid Earth, 2014. 119(9): p. 6924-6935.

6. Strom, A., Morphology and internal structure of rockslides and rock avalanches: grounds and constraints for their modelling, in Landslides from Massive Rock Slope Failure, S.G. Evan, et al., Editors. 2006, Springer Netherlands. p. 305-326.

360 7. Rait, K.L., E.T. Bowman, and C. Lambert, Dynamic fragmentation of rock clasts under normal compression in sturzstrom. Géotechnique Letters, 2012. 2(3): p. 167-172.

8. Qin, C. and S.C. Chian, Revisiting crown stability of tunnels deeply buried in non-uniform rock surrounds. Tunnelling and Underground Space Technology, 2018. 73: p. 154-161.

9. Fenton, G.A. and D.V. Griffiths, Risk assessment in geotechnical engineering. 2008, New Jersey: John Wiley and Sons.

10. Liu, Y., F.-H. Lee, S.-T. Quek, and M. Beer, Modified linear estimation method for generating multi-dimensional multi-variate Gaussian field in modelling material properties. Probabilistic Engineering Mechanics, 2014. 38: p. 42-53.

11. Liu, Y., L.Q. He, Y.J. Jiang, M.M. Sun, E.J. Chen, and F.-H. Lee, Effect of in situ water content variation on the spatial variation of strength of deep cement-mixed clay. Géotechnique, 2019. 69(5): p. 391-405.

12. Casagrande, D., O. Buzzi, A. Giacomini, C. Lambert, and G. Fenton, A New Stochastic Approach to Predict Peak and Residual Shear Strength of Natural Rock Discontinuities. Rock Mech Rock Eng, 2018. 51(1): p. 69-99.

13. Shen, W.G., T. Zhao, G.B. Crosta, and F. Dai, Analysis of impact-induced rock fragmentation using a discrete element approach. Int. J. Rock Mech. Min., 2017. 98: p. 33-38.

14. Potyondy, D.O. and P.A. Cundall, A bonded-particle model for rock. Int. J. Rock Mech. Min., 2004. 41(8): p. 1329-1364.

15. Shen, Z., M. Jiang, and C. Thornton, DEM simulation of bonded granular material. Part I: Contact model and application to cemented sand. Computers and Geotechnics, 2016. 75: p. 192-209.

16. Bono, J., G. McDowell, and D. Wanatowski, Investigating the micro mechanics of cemented sand using DEM. Int. J. Numer. Anal. Methods Geomech., 2015. 39(6): p. 655-675.

17. Nova, R., R. Castellanza, and C. Tamagnini, A constitutive model for bonded geomaterials subject to mechanical and/or chemical degradation. Int. J. Numer. Anal. Methods Geomech., 2003. 27(9): p. 705-732. 
18. Alassi, H.T. and R. Holt, Relating discrete element method parameters to rock properties using classical and micropolar elasticity theories. Int. J. Numer. Anal. Methods Geomech., 2012. 36(10): p. $1350-1367$.

19. Zhao, T. and G.B. Crosta, On the dynamic fragmentation and lubrication of coseismic landslides. J. Geophys. Res.: Solid Earth, 2018. 123(11): p. 9914-9932

20. Thornton, C., K.K. Yin, and M.J. Adams, Numerical simulation of the impact fracture and fragmentation of agglomerates. Journal of Physics D-Applied Physics, 1996. 29(2): p. 424-435.

21. Grady, D.E., Fragmentation of Solids under Impulsive Stress Loading. J. Geophys. Res.: Solid Earth, 1981. 86(B2): p. 1047-1054.

22. Weatherley, D., V. Boros, and W. Hancock, ESyS-Particle Tutorial and User's Guide Version 2.1. 2011, Brisbane: Earth Systems Science Computational Centre, The University of Queensland. 153.

23. Wang, Y., A new algorithm to model the dynamics of 3-D bonded rigid bodies with rotations. Acta Geotechnica, 2009. 4(2): p. 117-127.

24. Tonon, F., Analysis of single rock blocks for general failure modes under conservative and nonconservative forces. Int. J. Numer. Anal. Methods Geomech., 2007. 31(14): p. 1567-1608.

25. Hamilton, W.R., On quaternions; or on a new system of imaginaries in algebra. The London, Edinburgh, and Dublin Philosophical Magazine and Journal of Science, 1844. 25(163): p. 10-13.

26. Wang, Y., S. Abe, S. Latham, and P. Mora, Implementation of Particle-scale Rotation in the 3-D Lattice Solid Model. pure and applied geophysics, 2006. 163(9): p. 1769-1785.

27. Zhao, T., G.B. Crosta, S. Utili, and F.V. De Blasio, Investigation of rock fragmentation during rockfalls and rock avalanches via 3-D discrete element analyses. J. Geophys. Res. Earth Surf., 2017. 122(3): p. 678-695.

28. Zhao, T., B. Crosta, G. Dattola, and S. Utili, Dynamic Fragmentation of Jointed Rock Blocks During Rockslide-Avalanches: Insights From Discrete Element Analyses. J. Geophys. Res.: Solid Earth, 2018. 123(4): p. 3250-3269.

29. Liu, Y., F.-H. Lee, S.-T. Quek, E.J. Chen, and J.-T. Yi, Effect of spatial variation of strength and modulus on the lateral compression response of cement-admixed clay slab. Géotechnique, 2015. 65(10): p. 851-865.

30. Liu, X., F. Dai, R. Zhang, and J. Liu, Static and dynamic uniaxial compression tests on coal rock considering the bedding directivity. Environmental Earth Sciences, 2015. 73(10): p. 5933-5949.

31. Zhang, Q.B. and J. Zhao, A Review of Dynamic Experimental Techniques and Mechanical Behaviour of Rock Materials. Rock Mech Rock Eng, 2014. 47(4): p. 1411-1478.

32. Cai, M., P.K. Kaiser, Y. Tasaka, T. Maejima, H. Morioka, and M. Minami, Generalized crack initiation and crack damage stress thresholds of brittle rock masses near underground excavations. Int. J. Rock Mech. Min., 2004. 41(5): p. 833-847. 